\title{
An electrostatic lens system for the deceleration of high intensity heavy ion beams
}

\author{
F. W. Abdelsalam, M. M. Abdelrahman* and B. A. Soliman \\ Accelerators and Ion Sources Department, Nuclear Research Center, Atomic Energy Authority P. O. Box: 13759Inchas, \\ Atomic Energy, Cairo, Egypt.
}

Received 31 January, 2014; Accepted 2 April, 2014

\begin{abstract}
This work focuses on the design and implementation of an electrostatic lens system to produce highintensity, low-energy ion beams, by means of numerical simulation of the beam trajectories (SIMION 7). The lens design was optimized using the simulation data obtained with a performance characteristics of the electrostatic lens system. This lens system maintains good beam focal quality for the deceleration of heavy ion beams of argon ions of with space charge of $10 \mathrm{~mA}$. The beam emittance and beam diameter as a function of the gap width for a decel voltage of $45 \mathrm{kv}$ of a deceleration lens system for a singly charged argon ion trajectories were investigated. A gap width of $5 \mathrm{~mm}$ and a tube diameter of $26 \mathrm{~mm}$ were found to be the minimum values. Also, a minimum of $24 \mathrm{~mm}$ of inner tube diameter was obtained for the relation between the inner tube diameter and the beam diameter. Beam emittance and beam diameter as a function of the focusing points for a decel voltage of $45 \mathrm{kv}$ of a deceleration lens system for a singly charged argon ion trajectories was studied. A minimum of $455 \mathrm{~mm}$ was obtained at the outer exit of the deceleration lens system. While for the beam diameter, a minimum of $460 \mathrm{~mm}$ was deduced. It was found also that, a minimum of $V_{\text {decel }}=43 \mathrm{kV}$, deceleration voltage was obtained for the relation between the deceleration voltage and beam emittance. Also, a minimum of $V_{\text {decel }}=49 \mathrm{kV}$ was found for the relation between the decelration voltage and beam diameter.
\end{abstract}

Key words: Deceleration lens system and decel voltage, SIMION computer program, Beam emittance and beam radius.

\section{INTRODUCTION}

High-current ion beams are widely used for basic research and high technology applications, including for example, heavy ion fusion research, high current linear accelerators, spacecraft control systems, high dose ion implantation for material surface modification, and for surface cleaning and activation prior to film deposition (Goncharov, 2013). The advent of sophisticated computational techniques for simulating the actions of electric and magnetic fields on the motions of chargedparticle beams under their influence has greatly facilitated 
the design of low-aberration systems for use in ion acceleration and transport. These codes have been employed to design a variety of beam transport components, including lenses, magnets, steerers, deceleration/acceleration electrode systems, etc., and to simulate ion extraction from solid and plasma emitters. Among the computer codes for simulating the extraction of space-charge-dominated ion beams from plasma ion sources are those described according to Whealton and Whitson (1980), Whealton et al. (1988), Boers (1993), Spadtke (1983) and Becker and Herrmannsfeldt (1992). Electrostatic cylinder lenses are widely used to control beams of charged particles with different energies and directions in experiments covering many applications including electron spectroscopy, surface science and mass spectroscopy (Sise et al., 2007; 2005), and find use in focused ion beam systems, where they are more effective than magnetic lenses.

Electrostatic lenses usually consist of simple electrodes which are easy to manufacture and align. The major manufacturating problems are electric breakdown and the accumulation of charges on the insulating surfaces. The properties of the lenses comprising a pair of symmetrically arranged cylinders depend on a large number of parameters: the electrode voltage ratios, cylinder sizes and the gap between the electrodes. The field of electron and ion optics is based on the analogy between geometrical light optics and the motion of charged particles in electromagnetic fields. The aim is to concentrate as many particles in as small a volume as possible. The optical properties of electrostatic lenses are fixed by the voltage ratios. The focusing of charged particles with an electrostatic field can be achieved by devices consisting of a sequence of the accelerating electrodes with cylindrical symmetry which have lens like properties. Recently, a heavy ion injector was installed at the Accelerators and Ion Sources Department, Nuclear Research Center, the Atomic Energy Authority (A.E.A), Egypt (Abdelrahman, 2009). The system is intended for use in isotope separators and ion implantation, as well as for research in the physics and technology of ion sources and surface physics. It is complete with power supply, control, vacuum and cooling system. This source (Nikiforov et al., 1996; Wielunski et al., 2004) was supplied by Efremov Research Institute of Electro Physical Apparatus, Saint Petersburg, Russia.

In the source, the ions are generated through crossed electric and magnetic fields discharge plasmas. The discharge is formed between the thermo emission core cathode and cylindrical anode surrounding it. The electrons emitted by the hot cathode have high ionization efficiency. The ions are extracted from the discharge region through an emission slit in the anode. The system uses an acceleration/deceleration scheme to avoid compensating electrons being accelerated back into the source, thus destroying its rear side and leaving the ion beam uncompensated. The main beam extraction characteristics of this Freeman type ion source system were investigated with the SIMION computer program version 7, using singly charged argon ions with and without space charge (Abdelrahman and Abdelsalam, 2011).

It is the intent of this paper to discuss the computer beam optical properties for the deceleration lens system used to decelerate an ion beam extracted from a Freeman type ion source. Design and performance characteristics of the electrostatic lens system which maintains good beam focal quality for the deceleration of heavy ion beams of argon ions of space charge of 10 $\mathrm{mA}$, are described.

\section{CONSTRUCTION OF THE ION SOURCE}

Ions of various elements are generated in this version of a Freeman type ion source (Aitken, 2004) and accelerated in a four electrode acceleration/deceleration extraction system to obtain energies of up to $50 \mathrm{keV}$. The ion source and the accelerating/decelerating system are constructed as a single unit mounted around the vacuum chamber of the system.

It consists of a thermoemission tungsten cathode rod with $2 \mathrm{~mm}$ diameter, fixed inside a molybdenum anode by boron nitride insulators. The anode has an emission slit $40 \mathrm{~mm}$ in length and $0.5-2 \mathrm{~mm}$ wide, which is formed by two replaceable molybdenum plates mounted between the anode and the emission electrode, where the distance between the cathode and the exit slit is equal to $3.7 \mathrm{~mm}$. An auxiliary magnetic field of 100 gauss is maintained parallel to the axis of the cathode. The anode contains three holes for feeding with gas, liquid or solid materials. A schematic diagram of this ion source system is shown in Figure 1.

\section{EMITTANCE OF AN ION BEAM}

A charged particle beam is a group of particles possessing about the same kinetic energy and moving in about the same direction. The high kinetic energies and good directionality of charged particles in beams make them useful for various applications, such as, beam lithography for micro circuits, thin film technology, radiation processing of materials, and free electron lasers (Jr. Humphries, 1990). Beams extracted from different types of ion sources have to be transmitted without any loss. It is very important that the particles striking the target should have the parameters required for the target application. For beam transmission without loss of particles, the cross section of the beam must not exceed a given maximum value of a well defined point. To achieve this we introduce a quantity, the emittance (Elsaftawy, 2013) which is important for high quality beams and which is defined by the product of the width and the 


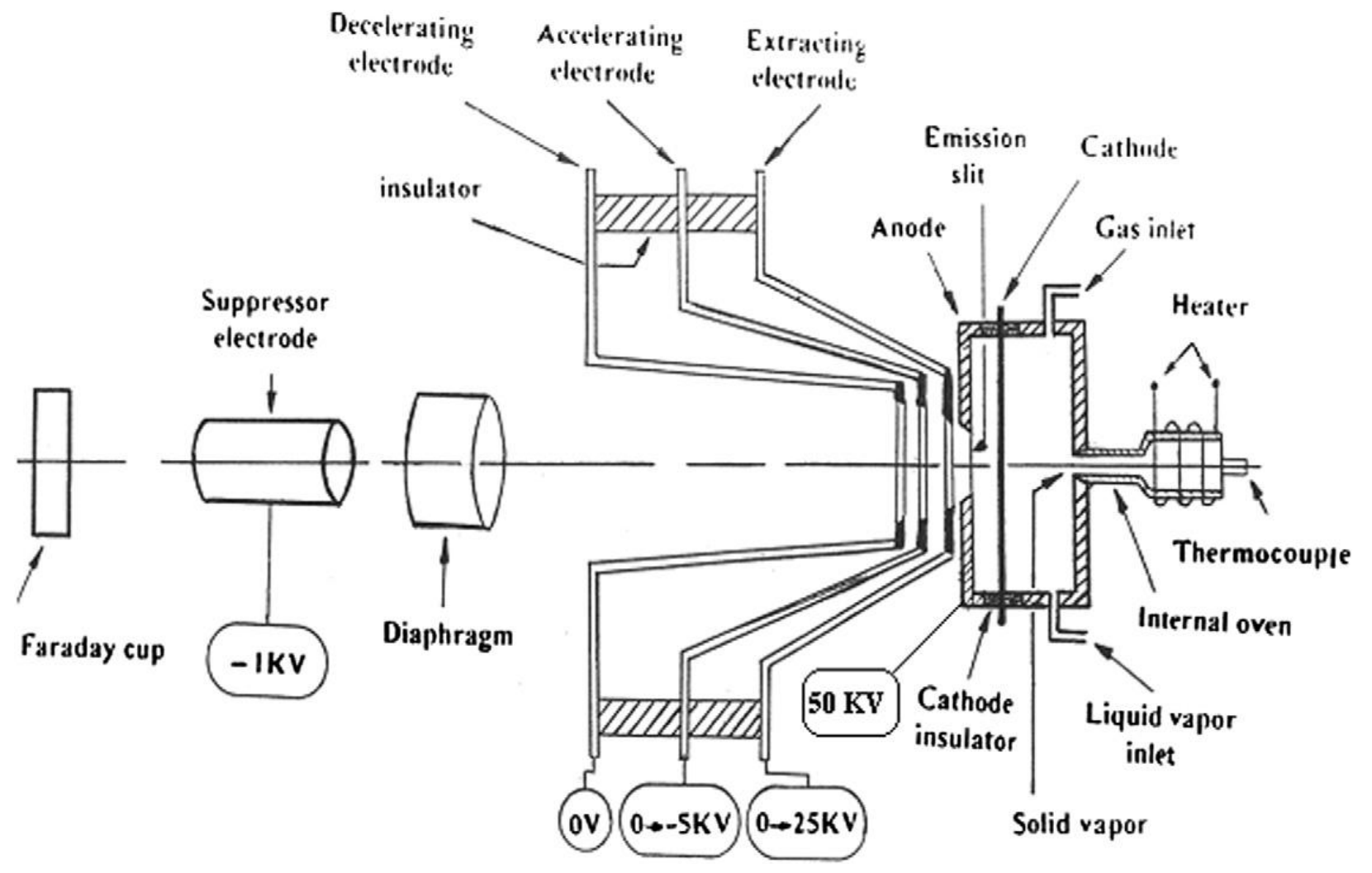

Figure 1. Schematic of the Freeman type ion source.

transverse velocity spread of the beam (region of phase space occupied by beam). If the beam is densely packed, then the emittance is said to be low and if it is somewhat spread out, it possesses high emittance (Masood et al., 2008). Any ion beam can be described in a six dimensional phase space $\left(x, y, z, p_{x}, p_{y}, p_{z}\right)$, where $(x, y$, $z)$ represents the position and $\left(p_{x}, p_{y}, p_{z}\right)$ the momentum components of the ions. The emittance of the beam, which must be invariant and independent of any electrostatic or magnetic fields through which the beam passes, is subject to Liouville's theorem (Forrester, 1988), which states that the motion of charged particles under the action of conservative fields is such that the local number density in six dimensional phase space is a conserved quantity. If a group of ions or electrons is accelerated through the same potential difference and travelling with small angles relative to the $z$ direction, we can say that they have a very small spread in $z$ directed velocities and that this spread is invariant. Then the phase space $\left(x, y, p_{x}, p_{y}\right)$ should be an invariant of the beam. For a beam with cylindrical symmetry, it implies that the two-dimensional area with coordinates $\left(r, p_{r}\right)$ should be an invariant. At a certain position on the $z$ axis (momentum component $p_{z}$ approximately equals the total particle momentum $P$ ), we consider ions which pass through $(x, y)$ related to $r$, and if cylindrical symmetry can be assumed (Abdelrahman and Zakhary, 2009) then:

$$
x^{\prime}=\frac{p_{x}}{p_{z}}=\frac{p_{x}}{p}, y^{\prime}=\frac{p_{y}}{p}, r^{\prime}=\frac{p_{r}}{p}
$$

Then,

$$
\varepsilon_{x}=\frac{1}{\pi} A\left(x, x^{\prime}\right), \varepsilon_{y}=\frac{1}{\pi} A\left(y, y^{\prime}\right), \varepsilon_{r}=\frac{1}{\pi} A\left(r, r^{\prime}\right)
$$

where $\varepsilon_{x}, \varepsilon_{y}$ and $\varepsilon_{r}$ are the transverse emittance or simply emittance in the $\left(x-x^{\prime}\right),\left(y-y^{\prime}\right)$ and $\left(r-r^{\prime}\right)$ planes respectively. The values $A\left(x, x^{\prime}\right), A\left(y, y^{\prime}\right)$ and $A\left(r, r^{\prime}\right)$ represent two dimensional phase space areas and remain constant along drift region (Figure 2).

The emittance is related to another property of accelerators or charged particle system namely the acceptance, sometimes called admittance. Acceptance is defined as the maximum emittance that a beam transport or analyzing system is able to transmit without flux loss. This is the size of the chamber transformed into phase space and does not suffer from the ambiguities of the definition of beam emittance. To get good performance of an ion beam transport system, the emittance should be much smaller than the acceptance (Lee, 1999). Figure 3 


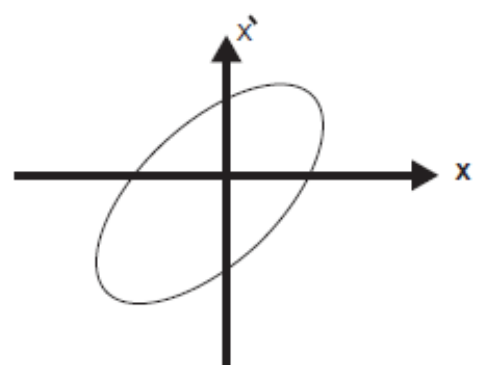

(a)

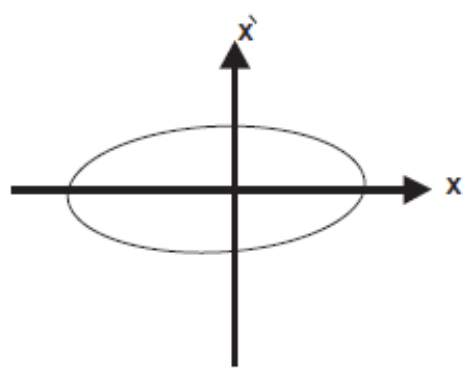

(c)

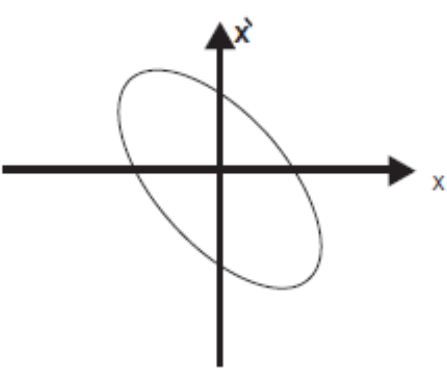

(b)

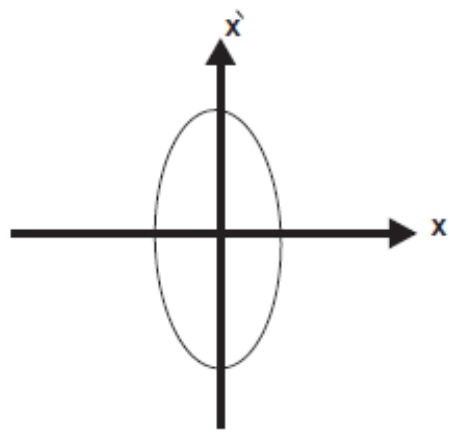

(d)

Figure 2. Different cases of two dimensional emittance patterns, with the corresponding beams as (a) divergent, (b) convergent, (c) parallel and (d) focused.

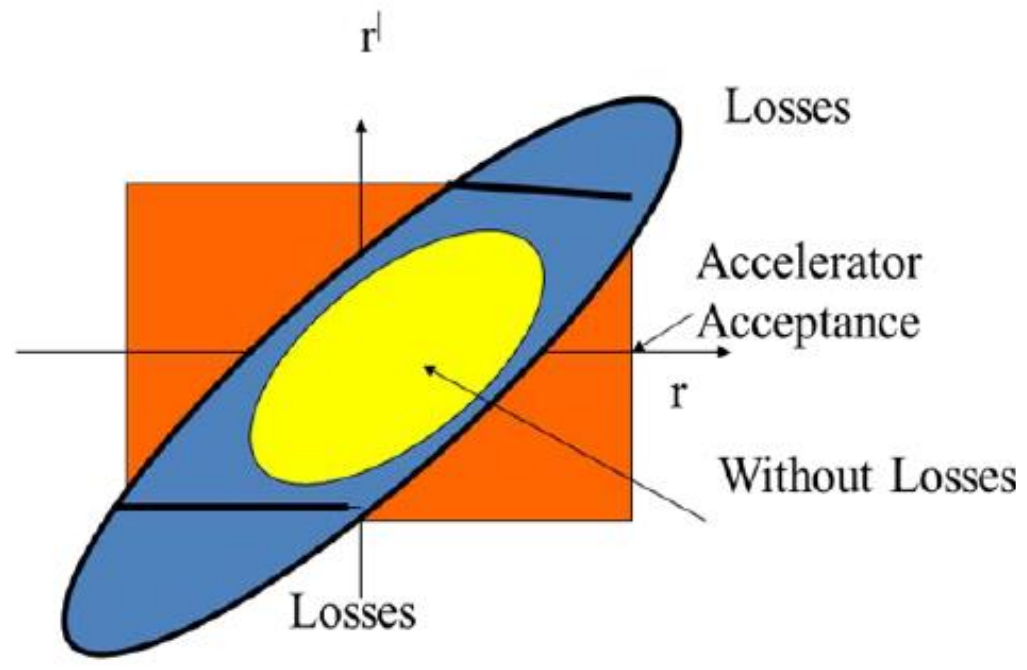

Figure 3. Accelerator acceptance with relation to the definition of emittance in phase space.

represents a schematic diagram that describes the acceptance of the accelerating system in phase space representation. To characterize the emittance of a beam in SIMION, it is necessary to create a user program, since the capability was not built into the original SIMION program. Each particle's coordinates and velocity components were tracked and logged. After the particles exited the simulation domain at the right of the mesh, their components were logged in a database, and the averages taken to find the emittance. 


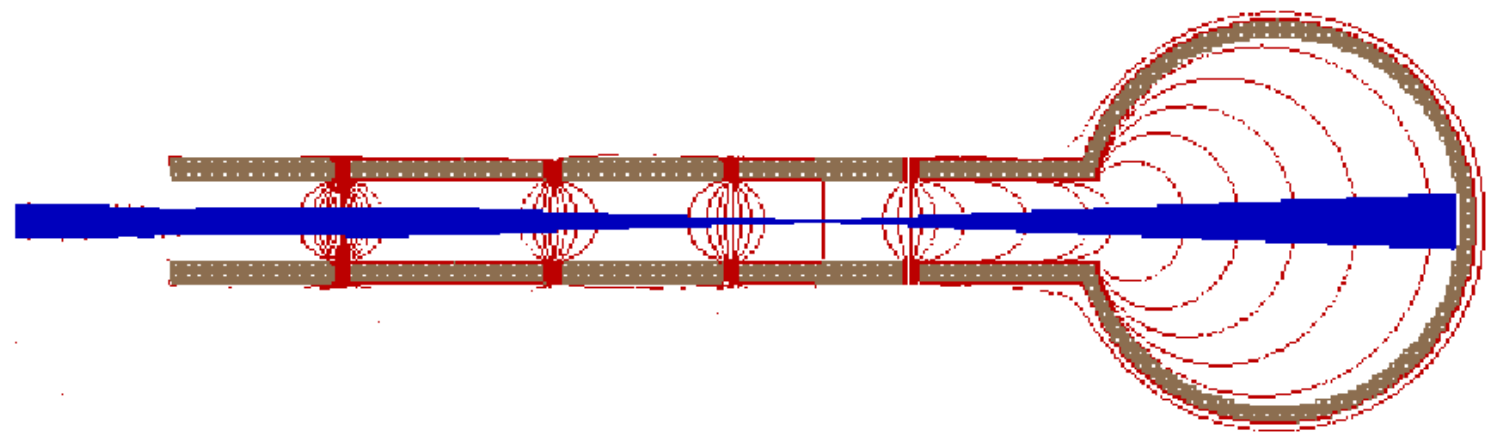

Figure 4. The Deceleration system with contours for singly charged argon ion trajectories.

In the present study, beam emittance was calculated by using the separate standard deviations of the positions $\left(\sigma_{y}\right)$ and the elevation angles $\left(\sigma_{\alpha}\right)$ for singly charged argon ion trajectories;

$\varepsilon=\sigma_{y} \cdot \sigma_{\alpha}$

Standard deviation can be defined as

$\sigma=\sqrt{\frac{1}{n-1} \sum_{i=1}^{n}\left(y_{i}-\bar{y}\right)^{2}}$

where $n$ is the number of repeated measurements, $y_{i}$ is the individual values and $\bar{y}$ is the mean value of the repeated-values. For a fixed geometry of the deceleration lens system, the following calculations have been done with space charge of $10 \mathrm{~mA}$.

\section{SIMULATION PROCESS}

The deceleration lens system was simulated using the ion optics simulation program SIMION (Dahl, 2000; Dahl et al., 1990). The program has been proven extremely useful in numerous applications. SIMION is an electrostatic lens analysis and design program that is capable of modeling charged particle optics problems with electrostatic and/or magnetic potential arrays. This program is used to simulate electrostatic and static magnetic devices for accelerating, transporting and otherwise manipulating beams of charged particles. For the purposes of this article, electrostatic fields only were modeled. In SIMION, five-point relaxation, a finite difference technique, is used as the iterative process to converge on the electric potential field solution for voltage contours and charged particle trajectories to be computed and plotted.
The lens system consists of a set of five cylindrical electrodes followed by a circle shaped electrode, all arranged along a common axis (Figure 4). As the beam traverses successive gaps between the electrodes, it encounters an increasing potential. The axial part of the electrostatic force is directed opposite to the direction of the incoming beam and has the net effect of reducing the energy of the beam.

Figure 5 shows the beam emittance and beam diameter as a function of the gap width for a decel voltage of $45 \mathrm{kv}$ of a deceleration lens system for singly charged argon ion trajectories. It is clear from the figure that, a minimum gap width of $5 \mathrm{~mm}$ was obtained for its relation to beam emittance while an increase of the gap width was accompanied by an increase in beam diameter. Beam emittance and beam diameter as a function of the tube diameter for a decel voltage of $45 \mathrm{kv}$ of a deceleration lens system for a singly charged argon ion trajectories was investigated (Figure 6). This figure shows that, there is a minimum of $26 \mathrm{~mm}$ of tube diameter for the relation between the inner tube diameter and beam emittance. Also, a minimum of $24 \mathrm{~mm}$ of inner tube diameter was obtained for the relation between the inner tube diameter and the beam diameter. Beam emittance and beam diameter for singly charged argon ion trajectories as a function of the focusing points for a decel voltage of $45 \mathrm{kv}$ of a deceleration lens system were studied (Figure 7). A minimum of $455 \mathrm{~mm}$ was obtained at the outer exit of the deceleration lens system for the relation between the beam emittance and focusing points. While for the relation between the focusing points and beam diameter, a minimum of $460 \mathrm{~mm}$ was deduced. Figure 8 shows the influence of the deceleration voltage on both the beam emittance and beam diameter of a deceleration lens system for singly charged argon ion trajectories. It is found from this figure that, a minimum deceleration voltage of $V_{\text {decel }}=43 \mathrm{kV}$, was obtained for the relation between the deceleration voltage and beam emittance. Also, a minimum of $\mathrm{V}_{\text {decel }}=49 \mathrm{kV}$ is found for the relation between the deceleration voltage and beam diameter. 

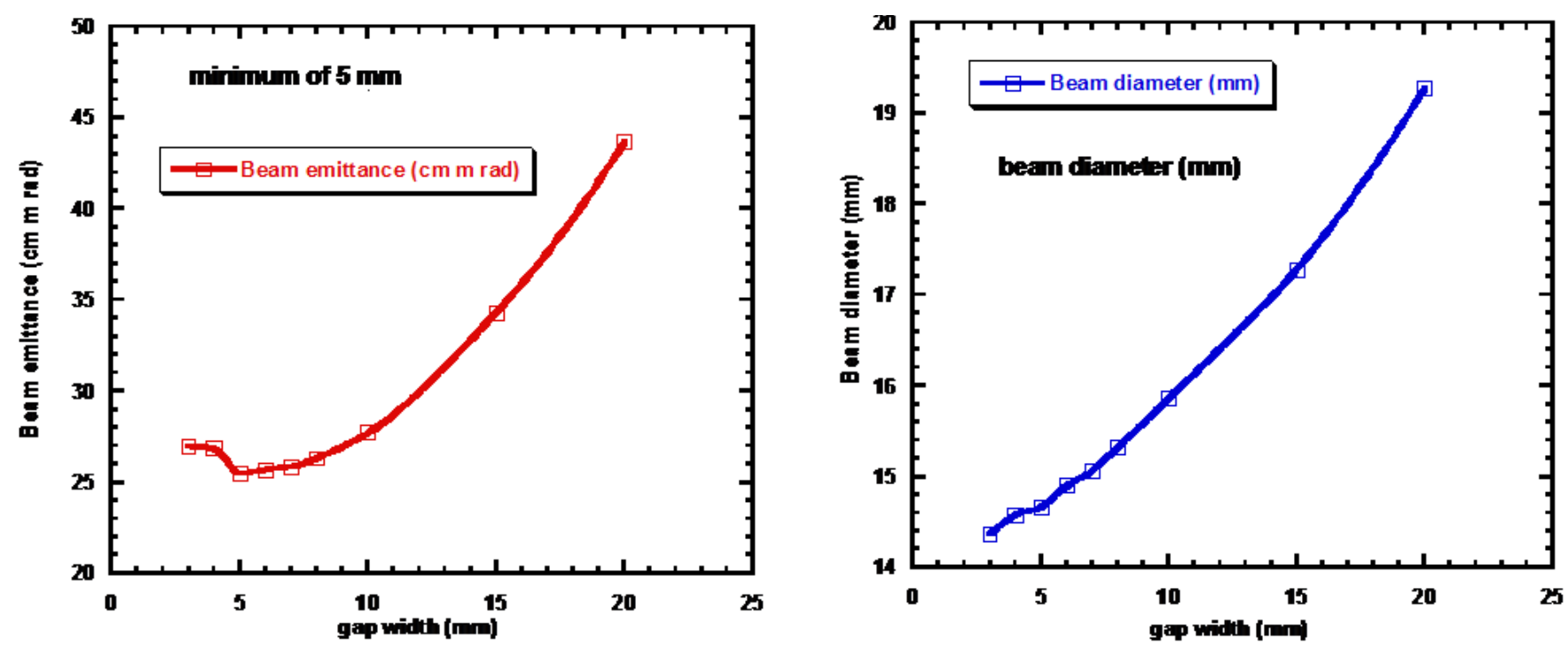

Figure 5. Beam emittance and beam diameter as a function of the gap width for a decel voltage of $45 \mathrm{kv}$ of a deceleration lens system for a singly charged argon ion trajectories.
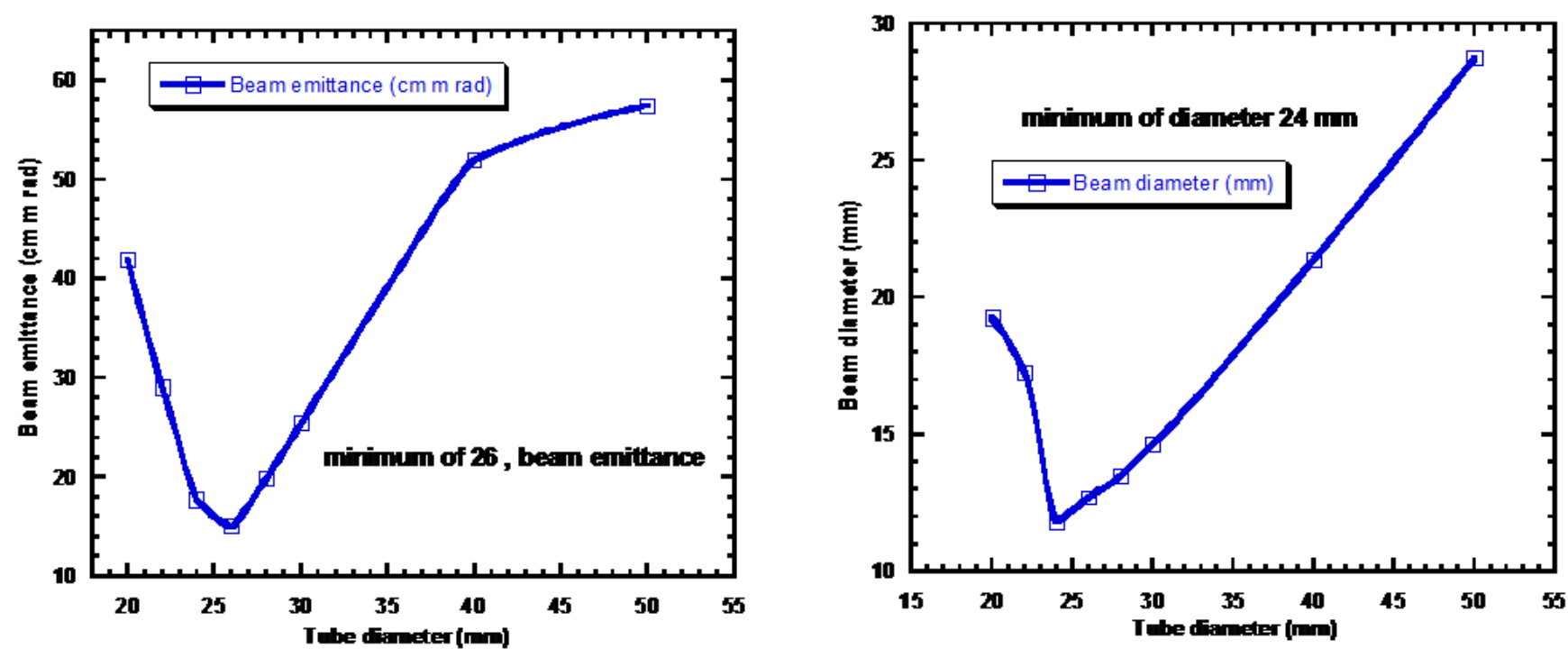

Figure 6. Beam emittance and beam diameter as a function of the tube diameter for a decel voltage of $45 \mathrm{kv}$ of a deceleration lens system for singly charged argon ion trajectories.

Figure 9 illustrates a study of the influence of deceleration voltage on both beam emittance and beam diameter of a deceleration lens system for singly charged argon ion trajectories at the center of the experimental chamber. It was deduced from this figure that, a minimum deceleration voltage, $\mathrm{V}_{\text {decel }}=47.5 \mathrm{kV}$ occurred for the relation between the deceleration voltage and beam emittance at the center of the experimental chamber. In case of the relation between the decelration voltage and beam diameter, increasing decelration voltage applied to the decelration electrode was accompanied by decreasing beam diameter.

\section{CONCLUSIONS}

These studies clearly demonstrate the necessity of providing means for varying the extraction gap to assure 

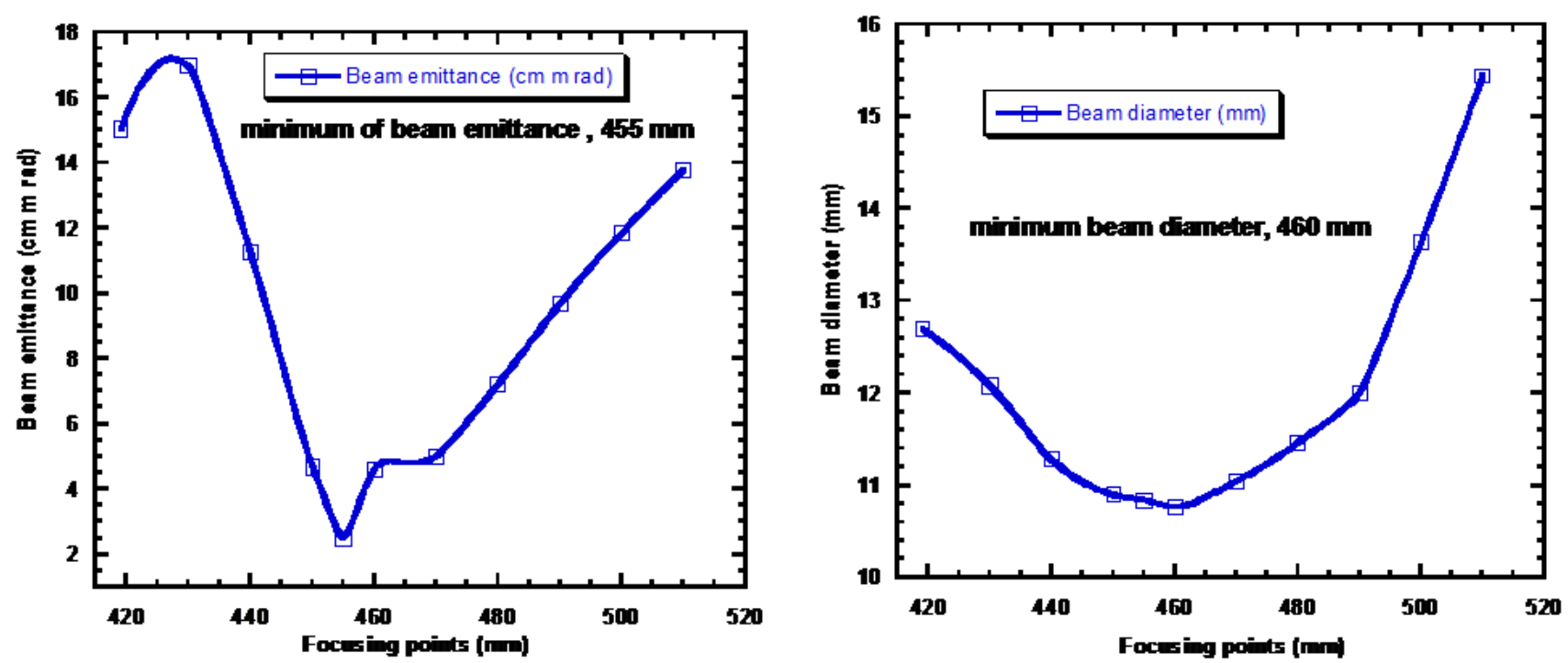

Figure 7. Beam emittance and beam diameter as a function of the focusing points for a decel voltage of $45 \mathrm{kv}$ of a deceleration lens system for singly charged argon ion trajectories.
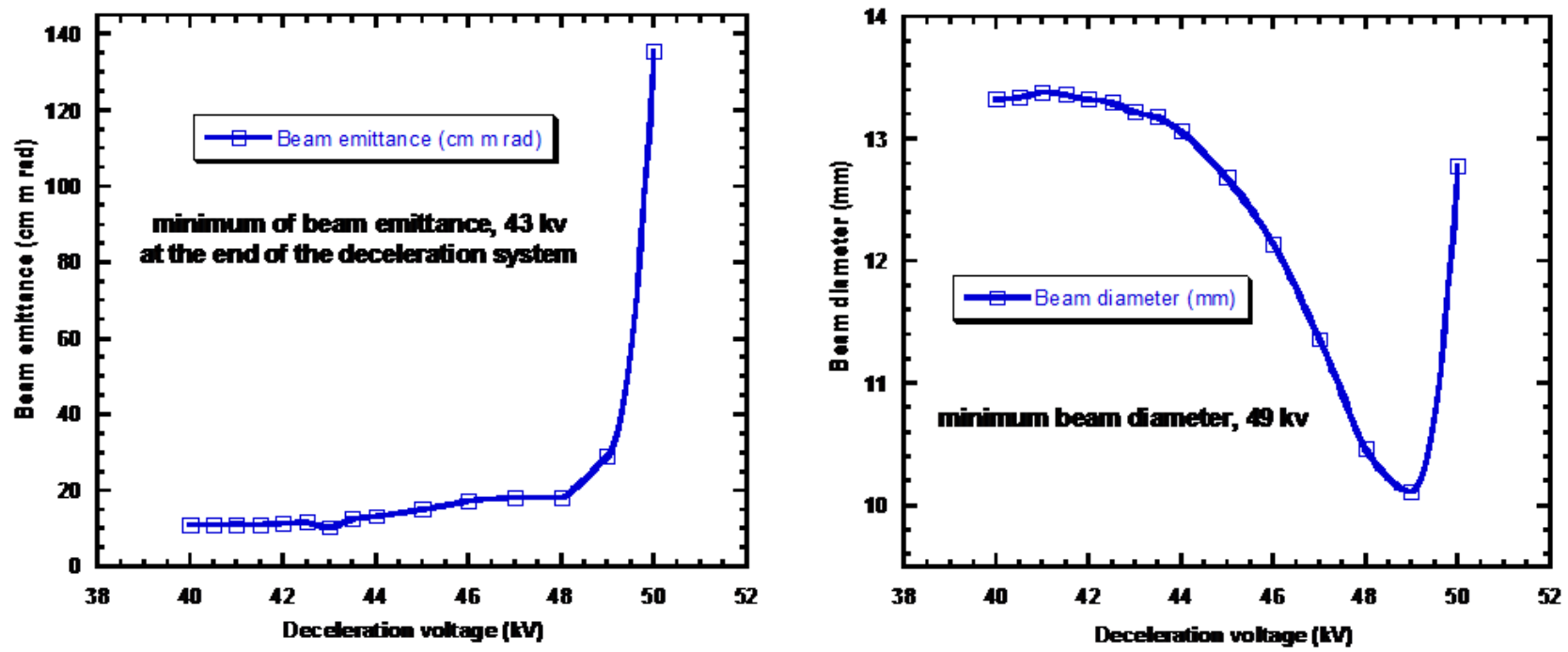

Figure 8. Influence of the deceleration voltage on both the beam emittance and the beam diameter of a deceleration lens system for singly charged argon ion trajectories.

high quality space-charge-dominated beams and also find the optimum parameters for the deceleration lens system which will be used experimentally in the next stage in isotope separation of ion beams of heavy ions. It was found that a minimum of gap width of $5 \mathrm{~mm}$ was obtained for the relation between the gap width and beam emittance while an increase of the gap width was accompanied by an increase of the beam diameter. Also, a minimum of $26 \mathrm{~mm}$ of tube diameter was obtained for the relation between the inner tube diameter and beam emittance. Furthermore, a minimum of $24 \mathrm{~mm}$ of inner tube diameter was obtained for the relation between the inner tube diameter and the beam diameter. In further consequence, a minimum of $455 \mathrm{~mm}$ was obtained at the outer exit of the deceleration lens system for the relation between the beam emittance and focusing points. While for the relation between the focusing points and beam diameter, a minimum of $460 \mathrm{~mm}$ was deduced. It was found that a minimum of deceleration voltage $V_{\text {decel }}=43$ $\mathrm{kV}$, was obtained for the relation between the 

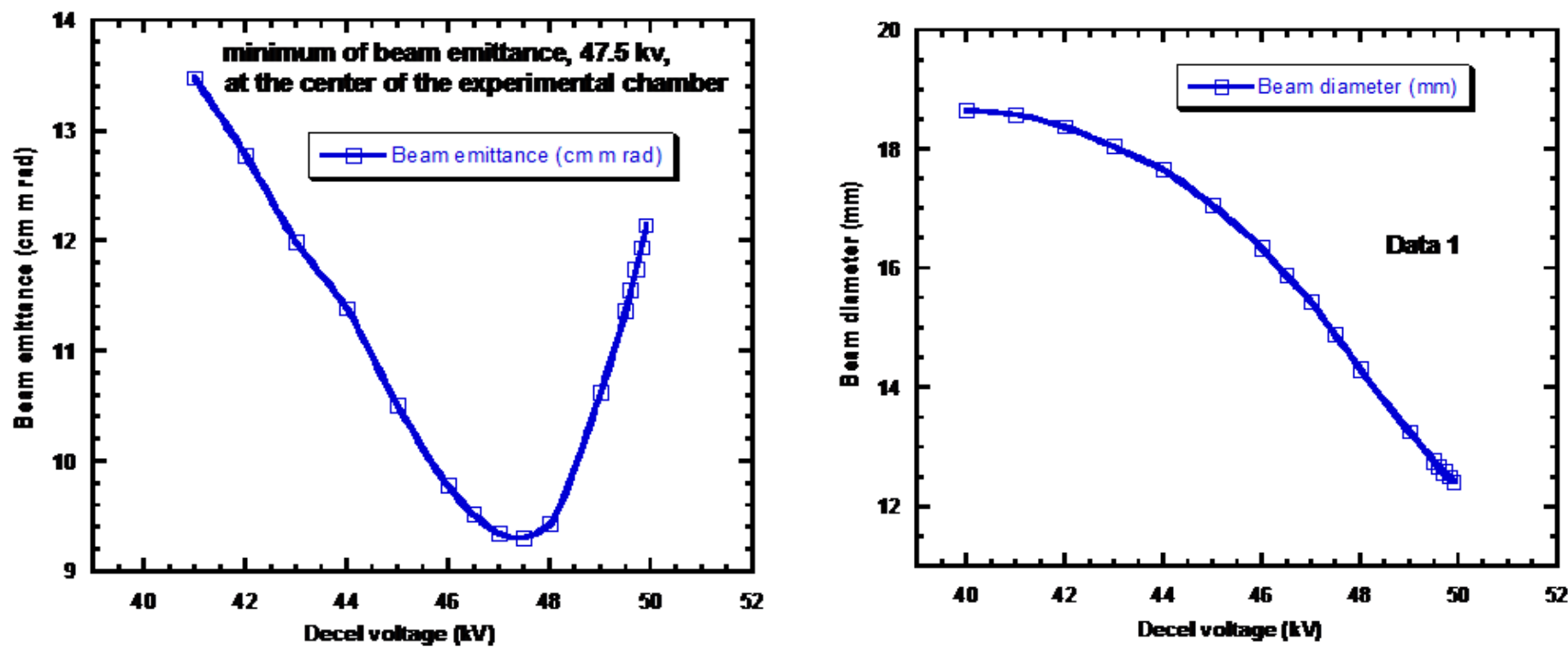

Figure 9. Influence of deceleration voltage on both the beam emittance and beam diameter of a deceleration lens system for singly charged argon ion trajectories at the center of the experimental chamber.

deceleration voltage and beam emittance. Also, a minimum, $V_{\text {decel }}=49 \mathrm{kV}$ was found for the relation between the deceleration voltage and beam diameter. Finally, a minimum of deceleration voltage, $\mathrm{V}_{\text {decel }}=47.5$ $\mathrm{kV}$ was obtained for the relation between the deceleration voltage and beam emittance. In case of the relation between the decelration voltage and beam diameter, an increase of the deceleration voltage applied to the deceleration electrode was accompanied by a decrease of the beam diameter. From these results, it was concluded that the ion beam extracted from this ion source was decelerated to a certain amount of energy with a newly designed and siumlated deceleration system for injection of an isotope separator system.

\section{Conflict of Interests}

The author(s) have not declared any conflict of interests

\section{REFERENCES}

Abdelrahman MM, El-Khabeary H (2009). Study of three different types of plasma ion sources. Plasma Sci. Technol. 11(5). http://dx.doi.org/10.1088/1009-0630/11/5/17

Abdelrahman MM, Abdelsalam FW (2011). Computer investigation of ion beam optics for a Freeman type ion source system. Nucl. instruments methods in phys. Res. B. 269:1761-1766. http://dx.doi.org/10.1016/j.nimb.2011.04.110

Abdelrahman MM, Zakhary SG (2009). Simulation studies for ion beam extraction systems. Brazilian J. Phy. 39:2. http://dx.doi.org/10.1590/S0103-97332009000300005

Aitken D (2004). In: I.G. Brown (Ed.). The physics and technology of ion sources, John Wiley, New York.
Becker R, Herrmannsfeldt WB (1992). IGUN-A program for the simulation of ion extraction, including magnetic fields. Rev. Sci. Instrum. 63:2756. http://dx.doi.org/10.1063/1.1142795, http://dx.doi.org/10.1063/1.1142795

Boers JE (1993). A digital computer code for the simulation of electron and ion beams on a PC, IEEE Cat. No. 93CH3334-0 213. (PBGUNS is electron/ion optics simulation code, developed by Thunderbird Simulations, Garland, TX, USA).

Dahl DA (2000). SIMION 3 D Version 7.0 User's Manual INEEL95/0403, Idaho National Engineering and Environmental Laboratory, I D 83415.

Dahl DA, Delmoreand JE, Appelhans AD (1990). SIMION PC/PS2 electrostatic lens design program. Rev. Sci. Instrum. 61:607. http://dx.doi.org/10.1103/PhysRevB.41.5763, http://dx.doi.org/10.1103/PhysRevB.41.5763

El-saftawy AM (2013). PhD. Thesis, Department of Physics Faculty of Science Zagazig University, Zagazig, Egypt.

Forrester AT (1988). "Large Ion Beams, Fundamental of Generation and Propagation "Wiley, New York. Humphries S Jr. (1990)."Charged Particle Beams", Wiley, New York.

Goncharov A (2013). The electrostatic plasma lens. Review of Scientific Instruments. 84:021101. http://dx.doi.org/10.1063/1.4789314, PMid:23464181, http://dx.doi.org/10.1063/1.4789314

Lee SY (1999). "Accelerator Physics", World Scientific, New York, USA.

Masood K, Iqbal M, Zakaullah M (2008). Emission characteristics of the thermionic electron beam sources developed at EBSDL. Nucl. Instrum. Meth Phys. Res. A 584:9. http://dx.doi.org/10.1016/j.nima.2007.09.049

Nikiforov S, Golubev V, Voronim G, Svinim M (1996). EPAC 96, Proceedings of the Fifth European Particle Accelerator Conference, Saint Petersburg.

Sise O, Ulu M, Dogan M (2005). Multi-element cylindrical electrostatic lens systems for focusing and controlling charged particles, Nucl. Instrum. Methods Phys. Res. A . $554: 114-131$.

Sise O, Ulu M, Dogan M (2007). Characterization and modeling of multielement electrostatic lens systems. Radiation Phys. Chem. 76:593598. http://dx.doi.org/10.1016/j.radphyschem.2005.11.037

Spadtke P (1983). AXCEL-GSI, Interaktives Simulations programm zur Berechnung von Ionbahnen in electrostatischen Feldern unter Berncksichtigung der Raumladung, GSI Report 9. 
Whealton JH, Bell MA, Raridon RJ, Rothe KE, Ryan PM (1988). Computer Modeling of Negative Ion Beam Formation. J. App Phys. http://dx.doi.org/10.1063/1.342078

Whealton JH, Whitson JC (1980). space-charge ion optics including extraction from a plasma, particle accelerators. 10:235-251.
Wielunski LS, Paterson GD, Bell JM, Clegg RE (2004). Production of $\mathrm{Si}_{+}$and $\mathrm{Cl}_{+}$ion beams from a Freeman type ion source using low toxicity and non-corrosive vapours as source gas Nucl. Instrum. Methods Phys. Res. B. 215:262-267. 